\title{
MANEJO DE DISFONÍA POST CIRUGÍA TIROIDEA CON LARINGOPLASTÍA POR INYECCIÓN CON ÁCIDO HIALURÓNICO*
}

\author{
Drs. Dahiana Pulgar B. ${ }^{1}$, Carla Napolitano V. ${ }^{2}$, Norma Inés León M. ${ }^{2}$, \\ Nicolás Dropplemann M. ${ }^{1}$, Pedro Badía V. ${ }^{2}$ \\ Departamento de Cirugía Oncológica. \\ 2 Departamento de Otorrinolaringología. Facultad de Medicina, Pontificia Universidad Católica de Chile. \\ Santiago, Chile.
}

\begin{abstract}
Hyaluronic acid in the management of post-thyroidectomy vocal cord paralysis

Introduction: Thyroid surgery rates have tripled over the past 3 decades. Currently, the main postoperative complications of this surgery are vocal fold paralysis because of recurrent laryngeal nerve dysfunction and hypocalcemia. Case report: We report the case of a 58 years old woman who presented with persistent dysphonia post total thyroidectomy. Laryngeal videostroboscopy evidenced unilateral vocal fold paralysis. We performed a percutaneous injection laryngoplasty with hyaluronic acid achieving significant improvement in voice. Conclusions: Percutaneous injection laryngoplasty with hyaluronic acid is a safe and effective procedure in the management of post-thyroidectomy vocal cord paralysis.
\end{abstract}

Key words: Hyaluronic acid, Injection laryngoplasty, Unilateral vocal fold paralysis.

\section{Resumen}

Introducción: La tasa de cirugía tiroidea se ha triplicado en las últimas 3 décadas. Dentro de sus complicaciones se encuentra la parálisis de cuerda vocal unilateral secundaria a lesión transitoria o permanente del nervio laríngeo recurrente, lo que se presenta clínicamente como disfonía. Caso clínico: Paciente de 58 años, post tiroidectomía total evoluciona con disfonía persistente. Videoestroboscopia evidencia parálisis cuerda vocal izquierda en posición paramediana, con hiato fonatorio longitudinal amplio. Se maneja con laringoplastía por inyección con ácido hialurónico a cuerda vocal paralítica, logrando mejora significativa de voz. En videoestroboscopia de control al $7^{\circ}$ día se evidencia cuerda vocal izquierda en línea media, con borde libre recto, sin presencia de hiato al fonar. Conclusiones: La laringoplastía por inyección percutánea con ácido hialurónico es un procedimiento seguro y eficaz para el tratamiento de la disfonía secundaria a parálisis cordal unilateral post cirugía tiroidea.

Palabras clave: Parálisis cuerda vocal, laringoplastía por inyección.

* Recibido el 30 de junio de 2014 y aceptado para publicación el 12 de agosto de 2014.

Los autores no refieren conflictos de interés.

Correspondencia: Dr. Pedro Badía V.

pbadia@med.puc.cl 


\section{Introducción}

La cirugía tiroidea se ha triplicado en Chile y el mundo durante las últimas décadas ${ }^{1}$. Dentro de sus complicaciones frecuentes se encuentra la lesión del nervio laríngeo recurrente (NLR) la cual puede ser permanente o transitoria ${ }^{2,3}$. Se ha descrito en distintas series una frecuencia de lesión del NLR permanente y transitoria del $0,3-3 \%$ y del $3-8 \%$ respectivamente ${ }^{4,5}$, siendo mayor en las resecciones extensas y en las re operaciones ${ }^{6}$. La lesión de NLR genera parálisis o paresia de cuerda vocal que puede manifestarse clínicamente con síntomas que van desde disfonía en las lesiones unilaterales hasta obstrucción de la vía aérea y estridor agudo en la parálisis bilateral ${ }^{7}$.

La disfonía secundaria a parálisis de cuerda vocal se origina por un cierre glótico incompleto que determina un escape de aire al fonar a este nivel, resultando en una voz soplada y débil. Además, la cuerda vocal paralizada con el tiempo presenta una disminución de su masa debido a atrofia del músculo tiroaritenoídeo, lo que acentúa la incompetencia glótica y la alteración vocal.

Se presenta un caso de disfonía secundaria a parálisis cordal unilateral post tiroidectomía total, tratada con laringoplastía por inyección percutánea con ácido hialurónico.

\section{Caso clínico}

Paciente de 58 años, sin antecedentes mórbidos de importancia. Se realiza tiroidectomía total por presencia de lesión folicular tiroidea en biopsia por punción de nódulo sospechoso. Biopsia definitiva informa hiperplasia uninodular folicular coloidea con zonas microfoliculares $(0,7 \times 0,5 \times 0,5 \mathrm{~cm})$. Evoluciona en post operatorio con disfonía persistente, sin estridor por lo que es derivada por tratante a comité de voz, siendo evaluada en conjunto por otorrinolaringología y fonoaudiología. Al examen destaca presencia de disfonía con voz soplada y débil, aumento del esfuerzo vocal, disminución de la resistencia vocal y fatiga vocal.

Se realiza videoestroboscopia laríngea que evidencia parálisis de cuerda vocal izquierda en posición paramediana, con hiato fonatorio longitudinal amplio. Mucosa cordal de aspecto normal sin lesiones focales. Onda mucosa conservada, de amplitud normal, asimétrica y aperiódica (Figura 1). Se realizó además valoración subjetiva de la discapacidad vocal, mediante la utilización del Voice Related Quality of Life (VRQOL) ${ }^{8}$, cuyo valor fue de $30 \%$ (considerado como pobre en la escala de calidad de vida).

Se decide realización de laringoplastía percutánea por inyección con ácido hialurónico para medializar y aumentar el volumen de la cuerda vocal izquierda con el objetivo de corregir el hiato fonatorio e insuficiencia glótica secundaria.

\section{Descripción del procedimiento}

Bajo técnica estéril se inyecta solución de lidocaína $2 \%$ + epinefrina $(0,025 \mathrm{mg} / \mathrm{ml})$ en zona de piel sobre membrana tirohioidea, cartílagos tiroideos y membrana cricotiroidea. Se administra además lidocaína intratraqueal $2 \mathrm{cc}$ al 4\%. Bajo visión nasofibrolaringoscópica, vía transtirohioidea se inyecta ácido hialurónico (Restylane ${ }^{\mathrm{TM}}$ ) en cuerda vocal
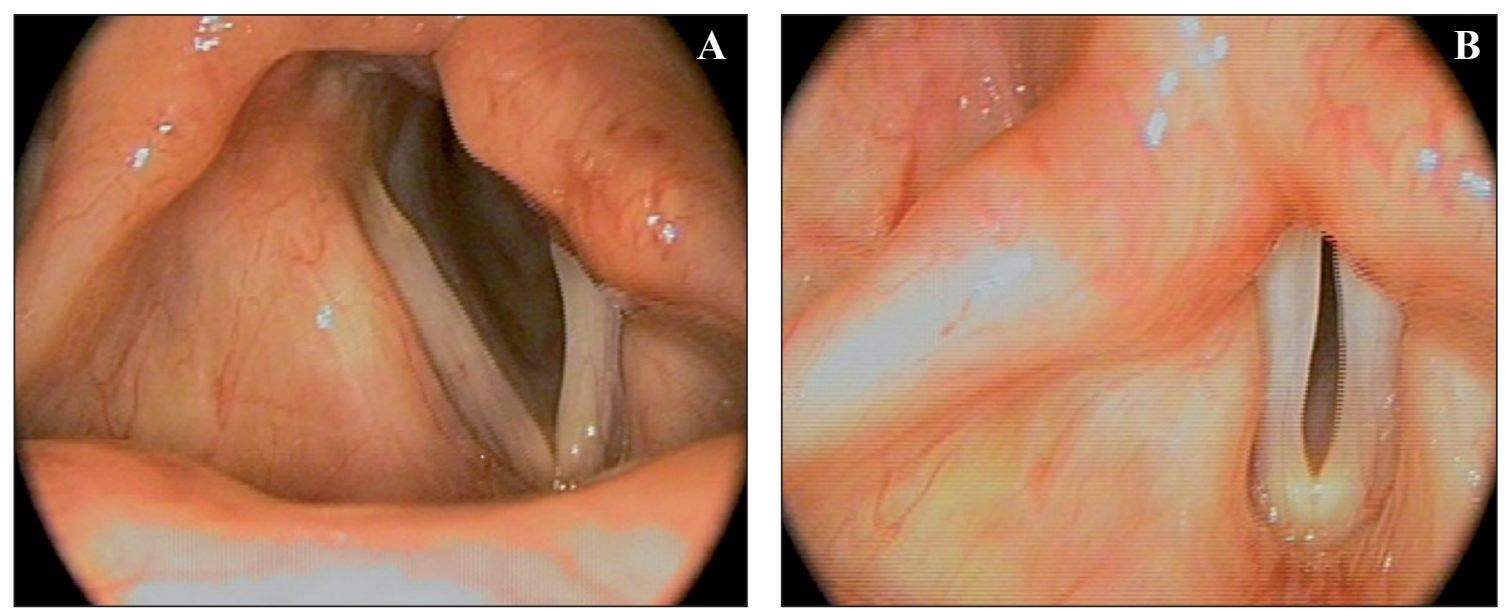

Figura 1. Imágenes de videoestroboscopia laríngea previo a la inyección de ácido hialurónico en cuerda vocal izquierda. A. Cuerda vocal izquierda en posición paramediana. B. Hiato fonatorio longitudinal amplio. 

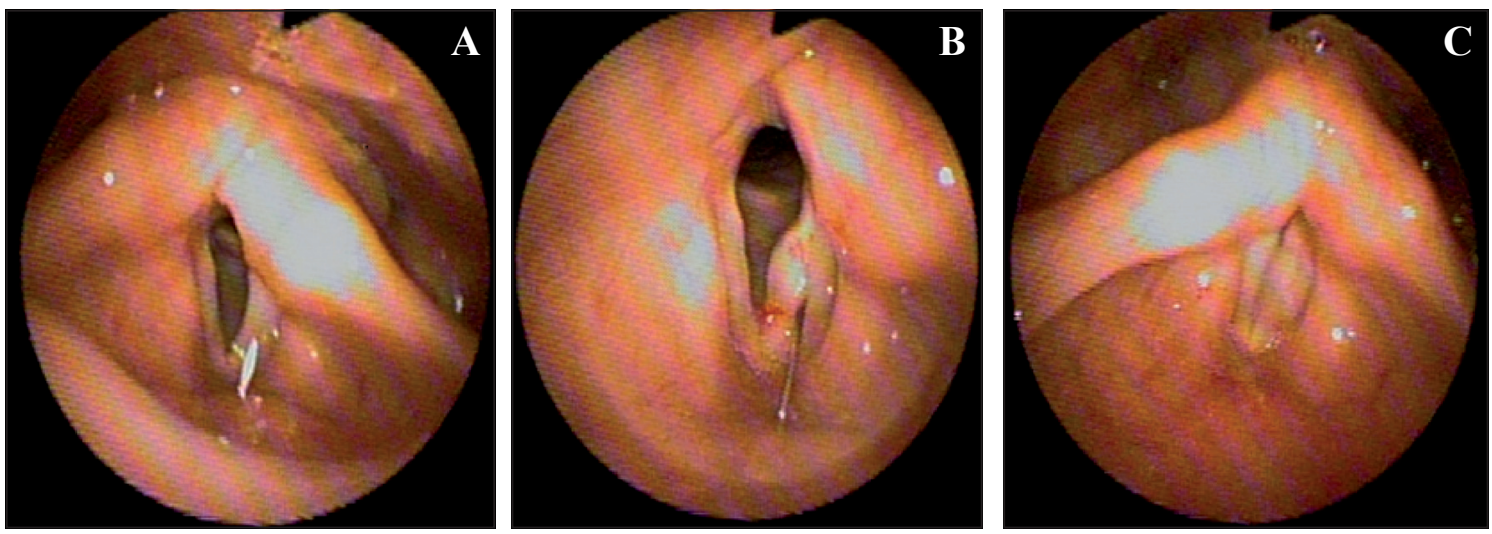

Figura 2. Imágenes de nasofibrolaringoscopia durante la inyección de ácido hialurónico en cuerda vocal izquierda. A. Cuerda vocal izquierda en posición paramediana y presencia de aguja a través del pecíolo de la epiglotis por un abordaje transtirohioideo. B. Administración de Restylane ${ }^{\mathrm{TM}}$ en cuerda vocal izquierda con aumento de volumen y medialización de ésta. C. Ausencia de hiato longitudinal al fonar posterior a inyección.

izquierda inmediatamente anterior a apófisis vocal y lateral al ligamento vocal (línea arcuata), hasta lograr mejoría en la calidad de la voz y corrección del hiato fonatorio ojival y de la incompetencia glótica. El procedimiento fue realizado sin complicaciones (Figura 2).

En control al $7^{\circ}$ día la paciente se presenta con buena voz, casi normal, y muy contenta con el resultado. En la videoestroboscopia laríngea de control se evidencia: cuerda vocal izquierda en línea media, con borde libre recto, sin presencia de hiato al fonar (Figura 3). La encuesta de VRQOL, demuestra una mejoría considerable, logrando un puntaje de $85 \%$ en la escala de calidad de vida. Paciente con evolución alejada favorable.

\section{Discusión}

Un porcentaje no despreciable de pacientes $(0$ al $87 \%$ ) evoluciona con disfonía post cirugía tiroidea y paratiroidea ${ }^{9}$. La principal causa de esta alteración vocal es la parálisis o paresia cordal unilateral secundaria a lesión de NLR transitoria o permanente 5 . La cuerda vocal paralizada tiende a adoptar una posición lateral o paramediana, lo que se traduce en insuficiencia glótica ${ }^{10}$. Estos pacientes requieren de un gran esfuerzo fonatorio, presentando voz débil y soplada, con presencia de fatiga vocal, lo que afecta de forma significativa el desempeño y calidad de vida del paciente, junto con retrasar la reincorporación al trabajo ${ }^{11}$.

La laringoplastía de medialización incluye a distintos procedimientos quirúrgicos que tienen como objetivo restaurar la posición anatómica de la cuerda vocal dentro de la laringe y solucionar la insuficiencia glótica. Se distinguen dos tipos de laringoplastía. La laringoplastía por inyección y la laringoplastía por vía externa o tiroplastía. La laringoplastía por inyección, descrita por Brunings en 1911 e implementada por Arnold en $1960^{12}$, consiste en la administración de distintos materiales en la cuerda vocal paralítica por vía transcutánea para generar un aumento de la masa y medialización de la misma, lo que mejora el cierre glótico ${ }^{13}$. Distintas sustancias han sido utilizadas como material de inyección, incluyendo teflón, colágeno bovino, colágeno autólogo, colágeno homólogo, hidroxiapatita de calcio y la grasa autóloga ${ }^{13}$. En el último tiempo, el ácido hialurónico (Restylane ${ }^{\mathrm{TM}}$ ) ha comenzado a utilizarse para laringoplastía por inyección. El ácido hialurónico es un polisacárido del grupo glucosaminoglicanos que forma parte de la matriz extracelular. Es uno de los componentes naturales del espacio de Reinke (compartimiento subepitelial de las cuerdas vocales). Su inyección en el músculo tiroaritenoideo genera un aumento del volumen del pliegue vocal, junto con empujarlo hacia medial y recrear la disposición tridimensional de éste. Además atrae a los fibroblastos, lo que genera nuevo colágeno y macromoléculas de la matriz extracelular, resultando en un aumento de tejidos blandos endógenos con un mínimo potencial inmunológico ${ }^{14}$.

Una guía clínica publicada el 2013, diseñada para mejorar los outcomes vocales posterior a cirugía tiroidea ${ }^{15}$, recomienda documentar cambios en la voz entre las 2 semanas y los 2 meses post cirugía tiroidea, valorando movilidad de cuerdas vocales. Ante la presencia de anormalidad, se sugiere derivación a otorrinolaringología para evaluación y 
manejo de disfunción vocal ${ }^{15}$. En el caso de nuestra paciente, esta fue enviada al mes post operatorio a evaluación por el comité de voz de nuestra institución, donde se realizó videoestroboscopia laríngea que evidenció una parálisis unilateral de cuerda vocal con presencia de hiato fonatorio amplio, por lo que se decidió tratamiento con laringoplastía por inyección percutánea con ácido hialurónico. Lee SW et $\mathrm{al}^{16}$, en un estudio que incluyó a 174 pacientes con insuficiencia glótica unilateral, (34 pacientes con post-tiroidectomía), manejados con laringoplastía por inyección concluyeron que este procedimiento mejora la voz y la calidad de vida en pacientes con parálisis de cuerda vocal unilateral post tiroidectomía, siendo un método simple, seguro y útil para su rehabilitación. En una revisión sistemática y metaanálisis reciente ${ }^{17}$ que incluyó a 39 estudios con un total de 1.798 pacientes con parálisis cordal post tiroidectomía, se recomienda uso de laringoplastía por inyección con material absorbible y entrenamiento de la voz, durante los primeros 12 meses post cirugía, como tratamiento en pacientes con parálisis cordal unilateral.

Si analizamos el pronóstico de recuperación de la voz, en un plazo promedio de 9 meses, de pacientes con parálisis cordal unilateral potencialmente recuperable sometidos a una laringoplastía de inyección como tratamiento temporal, observamos que el $72 \%$ recupera la voz y por lo tanto, no requiere un tratamiento definitivo (tiroplastía de medialización). De éstos, el 34\% lo hace con movilidad cordal parcial o total y el 38\% lo logra sin recuperación de movilidad cordal gracias a un reposicionamiento de la cuerda vocal en la línea media. Ahora si este mismo grupo de pacientes sólo lo observamos sin realizar ninguna intervención en el mismo período de 9 meses, el $67 \%$ de ellos no logra una voz adecuada y requiere una tiroplastía de medialización para corregir su voz ${ }^{18-20}$.

Comunicamos este caso por la alta frecuencia de disfonía post cirugía tiroidea. Sugerimos en estos pacientes una derivación precoz para estudio de movilidad cordal si presentan disfonía persistente por más de 2 semanas. Si bien no existe un tratamiento único para esta alteración, la laringoplastía por inyección de ácido hialurónico ha demostrado ser de utilidad y mejorar la calidad de vida de estos pacientes.

\section{Referencias}

1. Pellegriti G, Frasca F, Regalbuto C, Squatrito S, Vigneri R. Worldwide increasing incidence of thyroid cancer: update on epidemiology and risk factors. Journal of Cancer Epidemiology 2013;2013:965212.
2. Jeannon JP, Orabi AA, Bruch GA, Abdalsalam HA, Simo R. Diagnosis of recurrent laryngeal nerve palsy after thyroidectomy: a systematic review. International Journal of Clinical Practice. 2009;63:624-9.

3. Filho JG, Kowalski LP. Postoperative complications of thyroidectomy for differentiated thyroid carcinoma. American Journal of Otolaryngology 2004;25:225-30.

4. Lo CY, Kwok KF, Yuen PW. A prospective evaluation of recurrent laryngeal nerve paralysis during thyroidectomy. Arch Surg. 2000; 135:204-7.

5. Hayward NJ, Grodski S, Yeung M, Johnson WR, Serpell J. Recurrent laryngeal nerve injury in thyroid surgery: a review. ANZ Journal of Surgery 2013;83:15-21.

6. Thomusch O, Machens A, Sekulla C, Ukkat J, Lippert $\mathrm{H}$, Gastinger I, et al. Multivariate analysis of risk factors for postoperative complications in benign goiter surgery: prospective multicenter study in Germany. World Journal of Surgery 2000;24:1335-41.

7. Misiolek M, Waler J, Namyslowski G, Kucharzewski M, Podwinski A, Czecior E. Recurrent laryngeal nerve palsy after thyroid cancer surgery: a laryngological and surgical problem. Eur Arch Otorhinolaryngol. 2001;258:460-2.

8. Hogikyan ND, Sethuraman G. Validation of an instrument to measure voice-related quality of life (V-RQOL). Journal of Voice 1999;13:557-69.

9. Meek P, Carding PN, Howard DH, Lennard TW. Voice change following thyroid and parathyroid surgery. $\mathrm{J}$ Voice 2008;22:765-72.

10. Bielamowicz S, Stager SV. Diagnosis of unilateral recurrent laryngeal nerve paralysis: laryngeal electromyography, subjective rating scales, acoustic and aerodynamic measures. Laryngoscope 2006;116:359-64.

11. Szkielkowska A, Miaskiewicz B, Remacle M, Krasnodebska P, Skarzynski H. Quality of the voice after injection of hyaluronic acid into the vocal fold. Med Sci Monit. 2013;19:276-82.

12. Molteni G, Bergamini G, Ricci-Maccarini A, Marchese C, Ghidini A, Alicandri-Ciufelli M, et al. Autocrosslinked hyaluronan gel injections in phonosurgery. Otolaryngol Head Neck Surg. 2010;142:547-53.

13. O'Leary MA, Grillone GA. Injection laryngoplasty. Otolaryngol Clin North Am. 2006;39:43-54.

14. Perazzo PS, Duprat Ade C, Lancellotti CL. Histological behavior of the vocal fold after hyaluronic acid injection. J Voice 2009;23:95-8.

15. Chandrasekhar SS, Randolph GW, Seidman MD, Rosenfeld RM, Angelos P, Barkmeier-Kraemer J, et al. Clinical practice guideline: improving voice outcomes after thyroid surgery. Otolaryngol Head Neck Surg. 2013;148(6 Suppl):S1-37.

16. Lee SW, Kim JW, Chung CH, Mok JO, Shim SS, Koh $\mathrm{YW}$, et al. Utility of injection laryngoplasty in the management of post-thyroidectomy vocal cord paralysis. Thyroid 2010;20:513-7.

17. Chen X, Wan P, Yu Y, Li M, Xu Y, Huang P, et al. Types 
and Timing of Therapy for Vocal Fold Paresis/Paralysis After Thyroidectomy: A Systematic Review and MetaAnalysis. J Voice 2014;28:799-808.

18. Arviso LC, Johns MM, 3rd, Mathison CC, Klein AM. Long-term outcomes of injection laryngoplasty in patients with potentially recoverable vocal fold paralysis. Laryngoscope 2010;120:2237-40.

19. Yung KC, Likhterov I, Courey MS. Effect of temporary vocal fold injection medialization on the rate of permanent medialization laryngoplasty in unilateral vocal fold paralysis patients. Laryngoscope 2011;121:2191-4.

20. Prendes BL, Yung KC, Likhterov I, Schneider SL, AlJurf SA, Courey MS. Long-term effects of injection laryngoplasty with a temporary agent on voice quality and vocal fold position. Laryngoscope 2012;122:222733. 